\title{
MARCO LEGAL Y JURISPRUDENCIAL DEL ACOSO ESCOLAR EN COLOMBIA 10 AÑOS DE JURISPRUDENCIA CONSTITUCIONAL
}

José Alejandro Ruiz Forero* Recibido: Noviembre 1 de 2016 Aprobado: Diciembre 1 de 2016

\section{RESUMEN}

En este artículo se hace un análisis de la Jurisprudencia de la Corte Constitucional de Colombia que sirvió de base para el desarrollo legal del matoneo, censura o acoso escolar. Se hace una presentación de las sentencias más relevantes e importantes en las cuales la Corte decide casos de acoso escolar, así como de los motivos presentados ante el Congreso para la presentación del proyecto que terminaría convirtiéndose a la postre, en la Ley 1620 de 2013, también conocida como la Ley de Convivencia Escolar.

Palabras clave: acoso escolar, jurisprudencia, convivencia escolar, derechos constitucionales.

\section{LEGAL FRAMEWORK OF BULLYING IN COLOMBIA 10 YEARS OF CONSTITUTIONAL JURISPRUDENCE}

\begin{abstract}
This article does an analysis of the jurisprudence of the Constitutional Court that served as the base to the development of the legal framework of bullying in Colombia. It presents the most relevant and important cases in which the Court decides cases of school bullying, and it also

* Abogado, cursa Certificate IV in legal services en el College of Law Education and Training en Australia y es miembro de la organización Educación Diversa. Correo electrónico: educaciondiversa@gmail.com
\end{abstract}


refers to the arguments that motivated the moving forward of the bill that in turn became Law 1620 of 2013, also known as the Law of School Well-being.

Keywords: school bullying, precedent, School coexistence, Constitutional rights

\section{QUADRO JURÍDICO E JURISPRUDENCIAL DO ACOSSO ESCOLAR NA COLÔMBIA 10 ANOS DE JURISPRUDÊNCIA CONSTITUCIONAL}

\section{RESUMO}

Este artigo apresenta uma análise da jurisprudência do Tribunal Constitucional da Colômbia que foi a base para o desenvolvimento jurídico da intimidação, censura ou acosso escolar.

Se faz uma apresentação das sentenças mais relevantes e importantes em que o Tribunal decide casos de acosso escolar, assim como as razões apresentadas ao Congresso para a apresentação do projeto que acabaria por tornar-se, em última análise, a Lei 1620 de 2013, também conhecida como a lei da convivência escolar.

Palavras-chave: acosso escolar, jurisprudência, convivência escolar, direitos constitucionais.

\section{INTRODUCCIÓN}

El documento que se presenta a continuación, tiene como objetivo realizar un análisis legal y jurisprudencial del desarrollo del tema del acoso escolar o censura. Para lograr lo anterior, se realizó un proceso de investigación basado en las técnicas descritas por Diego Eduardo López Medina, en su obra "El Derecho de los Jueces". La investigación se centró específicamente en los 10 años comprendidos entre el 2006 y el 2016, ya que ha sido en este período durante el cual la Corte se ha pronunciado sobre este tema.

La metodología utilizada en el proceso de investigación, obliga a que se realice la lectura de las sentencias, de la más reciente a la más lejana, sin embargo, en el presente documento se analizará el proceso de manera cronológica, con el fin de intentar presentarle al lector la manera como el tema fue planteado en un comienzo, cómo se desarrolló y cuáles han sido 
las posiciones sostenidas por la Corte en sus pronunciamientos, así como del Congreso en el desarrollo de la Ley 1620 de 2013 también conocida como Ley de Convivencia Escolar.

Según Olweus citado por Castillo Pulido:

Un alumno es agredido o se convierte en víctima cuando está expuesto, de forma repetida y durante un tiempo, a acciones negativas que lleva a cabo otro alumno o varios de ellos. En esta situación se produce también un desequilibrio de fuerzas (una relación de poder asimétrica): el alumno expuesto a las acciones negativas tiene dificultad para defenderse y en cierto modo está desvalido frente a quienes lo hostigan" (2001, p. 419)

En ese orden de ideas, la Corte Constitucional considera que el acoso escolar puede causar la vulneración de múltiples derechos fundamentales reconocidos en la Constitución. La dignidad humana, el derecho a la intimidad, el derecho al libre desarrollo de la personalidad, entre otros.

Es indudable que la creación y el desarrollo del marco normativo sobre convivencia y acoso escolar en nuestro país, se debe en gran parte al esfuerzo realizado por la Corte Constitucional en su jurisprudencia. La ley y sus posteriores reglamentaciones han sido esfuerzos legislativos y ejecutivos por darle cumplimiento a las recomendaciones y órdenes impartidas por la corporación en sus sentencias. Sus pronunciamientos han sido argumentativamente sólidos y consistentes. Unicamente en dos de las sentencias seleccionadas se presentan aclaraciones al voto, ambas realizadas por el mismo Magistrado, el Dr. Jorge Ignacio Pretelt Chaljub. Las otras decisiones fueron adoptadas de manera unánime por la Corte, lo que da cuenta del consenso en la misma sobre la importancia del tema, así como la necesidad de preservar la unidad argumentativa y conceptual de la jurisprudencia constitucional.

Respecto a la Ley de 1620 de 2013, también conocida como Ley de Convivencia Escolar, es importante resaltar que se trató de un proyecto de ley cuyo trámite fue ampliamente respaldado por todas las fuerzas políticas representadas en el Congreso de la República. Tanto la entonces Ministra de Educación como dos de los tres ponentes del proyecto son miembros del Partido Conservador. El otro de los ponentes es miembro activo del Partido Liberal. En la página web de Congreso Visible (congresovisible.org) se puede consultar el trámite legislativo 
del proyecto, así como las votaciones a las que fue sometido durante el transcurso del mismo.

Resulta sorprendente, por decir lo menos, la reacción de sectores religiosos y conservadores, en torno a las iniciativas del Ministerio de Educación en cumplimiento de las órdenes impartidas por la Corte Constitucional sobre la necesidad de revisar y actualizar los manuales de convivencia de las Instituciones Educativas, con el fin de determinar que fueran respetuosos de la orientación sexual y la identidad de género, en aras de procurar la formación de ciudadanos respetuosos de los derechos humanos, sexuales y reproductivos, tal como lo pretendió la Ley de Convivencia Escolar concertada con las diversas fuerzas políticas del país, de las cuales ellos forman parte. Es desafortunado que el debate esté dominado por la desinformación, los prejuicios y la falta de conocimiento de lo consagrado en la normatividad, la jurisprudencia y los tratados internacionales de derechos humanos sobre la materia. La utilización de creencias religiosas es y será válida en la democracia, siempre que se basen en verdades científicas y no en simple interpretación de textos religiosos que no son vinculantes para la población en general, y que mucho menos reemplazan y derogan los postulados fundamentales del Estado Social y Democrático de Derecho diseñado en la Constitución de 1991.

Se hace evidente la imperiosa necesidad de implementar estrategias pedagógicas de diversa índole por parte de las Instituciones Educativas, con el fin de cumplir con las obligaciones que les fueron asignadas dentro del Sistema Nacional de Convivencia Escolar y Formación para los Derechos Humanos, la Educación para la Sexualidad y la Prevención y Mitigación de la Violencia Escolar. Es una verdadera tragedia que en este proceso se haya perdido la vida de por lo menos un joven. Lo es aún más el hecho de que el acoso al que se vio sometido proviniera de las directivas de la Institución Educativa de la que hacía parte, por el hecho de tener una orientación sexual diversa no ajustada a los parámetros de supuesta "normalidad" profesados por el Colegio.

Resulta lamentable también, ver cómo en varios de los casos analizados en el presente documento, la solución de los padres del menor que había sido víctima de acoso escolar o censura, fue retirarlo o retirarla de la Institución Educativa, y proceder a trasladarlo (a) a otra. Si bien este hecho no ha impedido que la Corte se pronuncie sobre el fondo de las controversias jurídicas planteadas en las acciones de tutela, eso pone en evidencia que los padres de quienes han sido afectados por tales 
actividades, no creen que sea posible una solución mediante procesos restaurativos que reparen los vínculos de la comunidad educativa afectada por las conductas o situaciones de acoso escolar o censura. Esto da cuenta de la falta de aplicación efectiva de los parámetros establecidos en la normatividad vigente, así como de la debilidad del Sistema de Inspección, Vigilancia y Control de las Entidades del sector.

Sin embargo, lo más preocupante es, en nuestra opinión, el desdén con el que muchas instituciones educativas, directivos, profesores y padres, tienen por los postulados, principios y valores consagrados en la Constitución, la jurisprudencia constitucional y la normatividad de convivencia escolar. Los colegios continúan incluyendo en sus manuales de convivencia estipulaciones que atentan y violan los derechos fundamentales de los estudiantes, como cuando establecen que determinada orientación sexual o demostraciones de afecto son consideradas inmorales o vulgares. Pareciera como si muchos colegios pusieran sus particulares confesiones religiosas y los textos teológicos que las soportan por encima de la Constitución Política que desde el año de 1991 rige en nuestro país. Así mismo, es sorprendente que con la amplia y numerosa jurisprudencia constitucional sobre los conflictos que se presentan entre derechos fundamentales de estudiantes y las instituciones educativas, como consecuencia de la aplicación de la normas contenidas en los Manuales de Convivencia, aún existan en dichos reglamentos, estipulaciones abiertamente discriminatorias, antidemocráticas y contrarias a los valores contenidos en la Carta Política.

\section{MARCO LEGAL Y JURISPRUDENCIAL DE LA CENSURA EN COLOMBIA. 10 AÑOS DE JURISPRUDENCIA CONSTITUCIONAL}

En lo que sigue se describirán los casos abordados por la jurisprudencia de Colombia sobre el tema y la incorporación de la Ley de convivencia escolar en esa línea.

Sentencia T-917 de 2006. Magistrado Ponente: Manuel José Cepeda Espinosa.

Unos estudiantes de noveno grado desvisten y filman a uno de sus compañeros durante una salida pedagógica, lo cual deriva en la iniciación de un proceso disciplinario en contra de cinco de ellos; en la posterior cancelación de su matrícula de la entidad educativa, y 
finalmente en la decisión de no renovación de las mismas luego de que sus derechos hubieran sido amparados durante el proceso de tutela, aduciendo que la decisión no se fundamentaba en el proceso disciplinario comenzado como resultado de los hechos mencionados, sino que se trataba de una decisión libre y consecuente con el principio de autonomía que la Ley le otorga a las instituciones educativas. Argumentó el Colegio que la decisión era el resultado de las continuas y constantes faltas disciplinarias de los estudiantes involucrados.

La corte plantea, entre varios, el siguiente problema jurídico: “ ¿Se vulneró el derecho a la dignidad del menor Santiago cuando fue perseguido en grupo, desvestido, ultrajado y filmado durante una salida pedagógica del colegio en el que cursaba el grado noveno?" Para resolver la citada cuestión constitucional, la Corte se pronuncia sobre dos cosas en particular: i) el derecho a la dignidad humana, a la autonomía y a la intimidad de los menores; y (ii) la relevancia de un proceso restaurativo, para resolver el caso concreto.

Para la Corte no queda duda de que se trata de una situación en la que los derechos fundamentales del menor, víctima de censura, fueron vulnerados, y establece que "La Constitución protege el derecho de un estudiante a no ser agredido por sus compañeros mediante la persecución en grupo para desvertirlo y filmarlo". En ese orden de ideas, la Corte señala que los derechos a la dignidad humana y la intimidad "protegen a los menores de la agresión de otros menores", por lo que el matoneo o acoso escolar es un atentado contra dichos derechos. La Corporación procede a realizar un análisis de estos en la jurisprudencia constitucional.

A continuación, la sentencia realiza una consideración que podemos considerar como la fundadora de la línea jurisprudencial propuesta, toda vez que para cumplir con la protección de los derechos a la dignidad, a la intimidad y a la autonomía de los menores, el presente caso no se puede circunscribir "a la garantía de un proceso disciplinario que sancione a los agresores sino también comprende la provisión de un proceso restaurativo que ofrezca una adecuada reparación a la víctima y restaure los vínculos de las partes con la comunidad”. En opinión de la Corte, un proceso disciplinario que pueda culminar con una sanción de los alumnos responsables de la situación de acoso escolar o censura,

puede en algunos casos ser insuficiente para asegurar el goce efectivos de los derechos constitucionales vulnerados por 
quienes cometieron la falta disciplinaria. Esto sucede cuando las consecuencias de la falta continúan perpetrándose de diversas maneras en el ámbito de la propia comunidad educativa. En tales eventos, la protección no formal sino real y efectiva de los derechos fundamentales lesionados exige medidas adicionales al proceso disciplinario. Corresponde a cada establecimiento educativo definir cuáles son las medidas adicionales aconsejables para lograr el objetivo tutelar de los derechos y, al mismo tiempo, para evitar que las secuelas de la lesión de dichos derechos se proyecte por distintas vías y continúe incidiendo negativamente en el ámbito de la comunidad educativa. Varias de esas medidas se pueden enmarcar en lo que se conoce como justicia restaurativa.

Según la Corte, los procesos de justicia restaurativa buscan "regenerar los vínculos sociales, psicológicos y relacionales de la víctima y el agresor con su comunidad", el resultado de un proceso de tipo restaurativo "comprende respuestas de arrepentimiento, perdón, restitución, responsabilización, rehabilitación y reinserción comunitaria, entre otros, que garanticen el restablecimiento de la dignidad de la víctima, su reparación y la restitución de los lazos existentes al interior de la comunidad". De esto se desprende que la "importancia de este tipo de procesos radica en que la falta no se concibe solo como una trasgresión de una norma, sino como un acontecimiento que afecta a la víctima, y repercute también en el agresor y en la comunidad".

A manera de conclusión la Corte le ordena al Colegio involucrado en el presente caso,

(...) que en el evento en que los tratos lesivos para la dignidad del menor víctima de los hechos se estén proyectando en su contra, como por ejemplo debido a la ventilación pública de los hechos, su estigmatización o la burla por parte de los miembros de la comunidad, deberá tomar medidas para que estos cesen. Dentro de estas medidas cabe adoptar algún tipo de proceso restaurativo a condición de que i) el menor afectado así lo acepte de manera autónoma, expresa e informada; y ii) alguno de los menores disciplinados vuelva a ser o haya seguido siendo parte de la comunidad educativa y acepte también participar en un proceso restaurativo. 
La importancia de esta sentencia radica en darles a las situaciones de acoso escolar o censura, un enfoque diferente al puramente disciplinario y sancionatorio, reconociéndose que debido a las particularidades propias de la vida escolar, es necesario además de adelantar procesos disciplinarios en situaciones como las presentadas, generar espacios y condiciones para que se den procesos restaurativos que de alguna manera redignifiquen tanto a la víctima como al agresor para que de esta manera se reparen los vínculos comunitarios lesionados. Esta línea de pensamiento es recogida nuevamente por la Corte en pronunciamientos posteriores, reiterándose la jurisprudencia en ella sentada.

Sentencia T-905 de 2011. Magistrado Ponente: Jorge Iván Palacio Palacio.

En este pronunciamiento, la Corte Constitucional hace por primera vez el esfuerzo de convocar a distintos actores expertos en el tema de la censura, matoneo o acoso escolar ${ }^{1}$. Esto denota un claro interés por parte de la Corte de analizar el tema a fondo y de realizar un pronunciamiento informado y soportado en información técnica y especializada que diera cuenta de la magnitud e importancia del fenómeno del acoso escolar o censura en nuestro país.

La Corte revisa una tutela presentada por los padres de una niña que ha sido objeto de burlas y ataques verbales por parte de sus compañeros de clase, haciendo referencia principalmente a su comportamiento y aspecto personal. El problema fue escalando, hasta que fue puesto en conocimiento de las autoridades escolares por los padres de la menor afectada, iniciándose un proceso que culminó con la inclusión de una anotación en el "observador del estudiante" de los menores involucrados en el despliegue de comportamientos y conductas constitutivas de acoso escolar, resultado que dejó inconformes a los accionantes.

La Corporación plantea el problema jurídico de la siguiente manera: “¿Vulneran los derechos fundamentales de una niña, los actos de

1 Es así como hace un resumen de las intervenciones de las siguientes personas: (i) Coordinadora del programa de licenciatura infantil de la Universidad del Norte; (ii) Decana (e) de la facultad de educación de la Universidad Autónoma de Bucaramanga; (iii) Jefe de la oficina asesora jurídica del Ministerio de Educación Nacional; (iv) Procuradora delegada para la defensa de los Derechos de la Infancia, Adolescencia y la Familia; (v) Profesora investigadora de la facultad de ciencias de la educación de la Universidad Externado de Colombia; (vi) Decano de la facultad de educación de la Pontificia Universidad Javeriana; (vii) Decano de la facultad de ciencias de la educación de la Universidad de la Salle; (viii) Decana de la facultad de ciencias de la educación de la Universidad Tecnológica de Pereira; (ix) Decana de la facultad educación de la Universidad Pedagógica Nacional; (x) Secretaría de Educación del Departamento; (xi) Oficina asesora jurídica de la Alcaldía Mayor de Bogotá; (xii) Defensor del Pueblo; y (xiii) Jefe de la oficina asesora jurídica del Instituto Colombiano de Bienestar Familiar. 
coerción y burla a los que es sometida por algunos de sus compañeros de clase, a pesar de haberse aplicado el respectivo manual de convivenciay de haberse impartido las sanciones correspondientes?"

Debido a que durante el proceso de tutela, los padres de la menor accionante deciden retirarla de la institución educativa en donde se desarrollaron los hechos y procedieron a matricularla en otra entidad, la Corte encuentra que en este caso

opera el fenómeno de la carencia actual de objeto, debido a que la razón por la cual se presentó la acción (el acoso escolar) ha desaparecido y no se detectan requerimientos que justifiquen la protección de los derechos de la menor (necesidad de asegurar que no sea hostigada por sus compañeros y que sea restaurada por los mismos y la comunidad que la rodeaba).

No obstante, la Corte entiende que al presentarse dicho fenómeno, no se cierra la posibilidad de que

esta Sala efectúe un análisis de las pruebas recaudadas y que tome decisiones en procura de garantizar los derechos de los niños y de las niñas que lleguen a encontrarse en circunstancias similares. En términos generales, de acuerdo con lo que fue respondido por las diferentes entidades estatales en todos los niveles y de los conceptos remitidos por varias universidades del país, se infiere que en la actualidad no existe una fórmula o herramienta coherente y efectiva que garantice la identificación y atención de los casos de acoso u hostigamiento escolar mediante un proceso restaurativo. (Negrilla fuera de texto).

En ese orden de ideas, la Corte entiende que si bien "no existe una pauta clara para definir en qué consiste la práctica del hostigamiento escolar o el "matoneo", se ha logrado evidenciar que los actos ejecutados por un grupo de compañeros en contra de K, (i) configuraron un desequilibrio entre los poderes o facultades de los estudiantes que, adicionalmente, (ii) constituyeron un acto de censura y rechazo ilegítimo e inconstitucional sobre aspectos personales de la niña y que (iii) terminaron por vulnerar su dignidad, en la medida en que la sometieron a un trato humillante". Así pues, según la Corte, "los acontecimientos que tuvo que soportar $\mathrm{K}$ no son el producto de actos inocentes, propios de la edad, o circunstanciales, sobre los cuales no había que prestar atención. En 
sentido estricto, los hechos denunciados por los padres de la menor, aunque no pueden encuadrarse como conductas criminales $u$ originadas en algún tipo de enfermedad -como fue mencionado por la Procuraduría General de la Nación- sí constituyen una forma de acoso u hostigamiento que debió ser prevenida, atendida y solucionada por la institución educativa y, si fuera del caso, por los demás sujetos y autoridades adscritas al esquema escolar y/o al Sistema Nacional de Bienestar Familiar, de manera que se materializaran el conjunto de obligaciones previstas en los artículos 38 y siguientes de la Ley 1098 de 2006”.

Como consecuencia del escenario planteado por la sentencia, para la Corte resultaba preocupante que no existiera "una definición de este fenómeno, sus elementos y tipologías o niveles de complejidad, de manera que sea posible distinguirla de otras formas de conflicto escolar; esto, obviamente, impide la diferenciación de una estrategia plena, que atienda las necesidades puntuales de las partes, los padres de familia y, adicionalmente, de los profesores". Es así como esta Corporación le ordena "al Ministerio de Educación, en coordinación con el Instituto Colombiano de Bienestar Familiar, la Defensoría del Pueblo y la Procuraduría General de la Nación, que lidere la formulación de una política general que permita la prevención, la detección y la atención de las prácticas de hostigamiento, acoso o matoneo escolar, de manera que sea coherente con los programas que se adelantan en la actualidad, con las competencias de las entidades territoriales y que constituya una herramienta básica para la actualización de todos los manuales de convivencia. Para tal efecto, se dispondrá el término de seis meses" (Negrilla fuera de texto).

Trámite Legislativo y análisis de la Ley 1620 de 2013 o Ley de Convivencia Escolar ${ }^{2}$.

El 8 de mayo de 2012 se radica el Informe de Ponencia para Primer Debate del Proyecto de Ley 201 de 2012 de la Cámara de Representantes, "por la cual se crea el Sistema Nacional de Convivencia Escolar y Formación para el Ejercicio de los Derechos Humanos, Sexuales y Reproductivos y la Prevención y Mitigación de la Violencia Escolar", y que a la postre se terminaría convirtiendo en la Ley 1620 de 2013. El proyecto fue presentado por la entonces Ministra de Educación Nacional, Dra. Maria Fernanda Campo, conjuntamente con un grupo de

2 El trámite legislativo de lo que se convirtió en la Ley de Convivencia Escolar, puede ser consultado en la siguiente página web: http://congresovisible.org/proyectos-de-ley/por-la-cual-se-crea-el-sistema-nacionalde-convivencia-escolar-y-formacion-para-el-ejercicio-de-los-derechos-humanos-sexuales-yreproductivos-y-la-prevencion-y-mitigacion-de-la-violencia-escolar-violencia-escolar/6634/ 
Representantes, "liderados por Juana Carolina Londoño Jaramillo, Telésforo Pedraza Ortega y Simón Gaviria Muñoz”. Para la elaboración del proyecto de ley, se recibieron los aporte e intervenciones de la Vicepresidencia de la República, de la Alta Consejería Presidencial para la Equidad de la Mujer, del Ministerio de la Protección Social, del Ministerio de Tecnologías de la Información y las Comunicaciones, del Instituto Colombiano de Bienestar Familiar, de la Policía Nacional y de la Oficina de la Alta Consejería de las Naciones Unidas para los Derechos Humanos (OACNUDH).

Según la ponencia, la propuesta legislativa buscaba recoger "los intereses de diversos sectores que han identificado la necesidad de fortalecer la convivencia escolar, la formación para el ejercicio de los Derechos Humanos, sexuales y reproductivos, la prevención y mitigación de la violencia escolar y el matoneo, tenido (sic) como objetivo fundamental la promoción de los Derechos Humanos y el mejoramiento de la convivencia en los establecimientos educativos públicos y privados" reconociéndose que, según estudios internacionales

el clima escolar es la variable que mayor influencia ejerce sobre el rendimiento de los estudiantes", el cual se define como el conjunto de "actitudes, creencias, valoraciones y normas que subyacen a las prácticas educativas, los logros académicos y las actividades propias de la escuela". En ese orden de ideas, se afirma que "cuanto mayor es la calidad académica de la escuela, menos es el nivel de delito y delincuencia de sus integrantes (...) además que en los ambientes escolares donde se presenta menor ocurrencia de vandalismo, violencia física o verbal entre el alumnado y menor discriminación por razones raciales, lingüísticas o sociales, los estudiantes tienden a obtener mejores resultados en sus procesos de aprendizaje.

De conformidad con la ponencia, en Colombia las prácticas de acoso escolar o bullying son más frecuentes en las edades comprendidas entre los 12 y los 14 años "es decir este tipo de violencia se presenta más frecuentemente en los años de educación básica (Grados 6, 7 y 8), la muestra más común de maltrato es la verbal y es a la que más miedo le tienen los escolares. También hay agresiones físicas y de exclusión. Además de golpes, burlas, chantajes y discriminación, los niños y jóvenes utilizan el ciberespacio, las redes sociales y demás herramientas tecnológicas digitales interactivas, como herramientas para el acoso 
escolar (Internet, telefonía móvil y video juegos online)". Posteriormente se hace una exposición de las características propias de esta nueva forma de acoso ligado a la era tecnológica ${ }^{3}$, y se señala que el fenómeno del matoneo o censura no se presenta exclusivamente en los estratos bajos, sino que se trata de una práctica generalizada en la sociedad, sin importar la condición socioeconómica de la víctima o del agresor.

Luego de citar una serie de cifras recogidas en las pruebas SABER del año 2005, así como un estudio exploratorio sobre el problema realizado por la Universidad Javeriana de la ciudad de Cali, la Encuesta Nacional de Deserción Escolar, entre otros, la ponencia concluye que si bien

se han dado pasos importantes en la construcción de marcos conceptuales, pedagógicos y operativos, acordes con la realidad del sector y con los diversos contextos, es importante establecer un marco normativo específico que desarrolle el principio constitucional de la responsabilidad compartida de instituciones educativas, familia, sociedad y Estado en la formación para la ciudadanía; defina sus funciones y facilite la aplicación de políticas intersectoriales donde la cooperación entre los diferentes actores sea el principio de acción y la herramienta para complementar y enriquecer la labor en la institución educativa" (Negrilla fuera de texto).

Como resultado de ese esfuerzo legislativo fue expedida la Ley 1620 de 2013, también conocida como la Ley de Convivencia Escolar, la cual en el artículo primero establece su objeto de "contribuir a la formación de ciudadanos activos que aporten a la construcción de una sociedad democrática, participativa, pluralista e intercultural" lo cual se logrará

\footnotetext{
3 "1. El ciberespacio es un entorno de socialización que además de tener identidad propia (es en Sí mismo un ¿lugar?), es transversal al resto de espacios: familia, escuela y comunidad. 2. Acosador y víctima no tienen siquiera que conocerse y las situaciones de bullying y ciberbullying no van siempre ligadas. Muchas veces es cierto que el acoso escolar se complementa con acecho virtual, pero no necesariamente. Puede incluso que un un conflicto online entre compañeros derive en bullying al trasladarse al otro espacio que partes comparten: el Establecimiento Educativo.

3. No hace falta ser fuerte para acosar en el espacio virtual. Tampoco quien sea poco sociable o tenga alguna característica que lo diferencia de su grupo de iguales, se convierte en víctima potencial de ciberacoso. Muy al contrario, ocurre en ocasiones que quien sufre bullying se transforma en acosador en el ciberespacio.

4. El cibberbullying según Enrique Chaux, tiene varios agravantes, como la permanencia e inasistencia en el acoso, ahora es de 24 horas. ¿Antes cuando un joven era intimidado en el colegio, al menos descansaba mientras no estaba en él? (sic), ahora se evidencia la propagación y persistencia total del acoso.

5. El Ciberbullying según los expertos, multiplica los efectos del acoso escolar tradicional, ya que expone a la víctima a escenarios online de gran aforo, en donde decenas de personas pueden animarse a decir cualquier cosa, lo que implica a la publicidad de la agresión".
} 
mediante el establecimiento del "Sistema Nacional de Convivencia Escolar y Formación para los Derechos Humanos, la Educación para la Sexualidad y la Prevención y Mitigación de la Violencia Escolar" con el fin de promover y fortalecer "la formación ciudadana y el ejercicio de los derechos humanos, sexuales y reproductivos de los estudiantes, de los niveles educativos de preescolar, básica y media y prevenga y mitigue la violencia escolar y el embarazo en la adolescencia”.

El artículo 2 de la misma ley, define el acoso escolar o censura y la cibercensura de la siguiente manera:

- Acoso escolar o bullying: Conducta negativa, intencional metódica y sistemática de agresión, intimidación, humillación, ridiculización, difamación, coacción, aislamiento deliberado, amenaza o incitación a la violencia o cualquier forma de maltrato psicológico, verbal, físico o por medios electrónicos contra un niño, niña, o adolescente, por parte de un estudiante o varios de sus pares con quienes mantiene una relación de poder asimétrica, que se presenta de forma reiterada o a lo largo de un tiempo determinado.

También puede ocurrir por parte de docentes contra estudiantes, o por parte de estudiantes contra docentes, ante la indiferencia o complicidad de su entorno. El acoso escolar tiene consecuencias sobre la salud, el bienestar emocional y el rendimiento escolar de los estudiantes y sobre el ambiente de aprendizaje y el clima escolar del establecimiento educativo".

Ciberbullying o ciberacoso escolar: Forma de intimidación con uso deliberado de tecnologías de información (internet, redes sociales virtuales, telefonía móvil y videojuegos online) para ejercer maltrato psicológico y continuado".

Realizar una análisis detallado del Sistema desborda el objetivo del presente trabajo. Sin embargo se referenciarán las contribuciones que consideramos más importantes de la Ley, en especial aquellas que hacen referencia a la prevención o mitigación de formas de violencia escolar. Así las cosas, dentro de los objetivos del Sistema Nacional de Convivencia Escolar y Formación para los Derechos Humanos, la Educación para la Sexualidad y la Prevención y Mitigación de la Violencia Escolar, se señalan entre otros, en su artículo cuarto: 
4. Promover el desarrollo de estrategias, programas y actividades para que las entidades en los diferentes niveles del Sistema y los establecimientos educativos fortalezcan la ciudadanía activa y la convivencia pacífica, la promoción de derechos y estilos de vida saludable (sic), la prevención, detección, atención y seguimiento de los casos de violencia escolar, acoso escolar o vulneración de derechos sexuales y reproductivos e incidir en la prevención y mitigación de los mismos, en la reducción del embarazo precoz de adolescentes y en el mejoramiento del clima escolar.

5. Fomentar mecanismos de prevención, protección, detección temprana y denuncia de todas aquellas conductas que atentan contra la convivencia escolar, la ciudadanía y el ejercicio de los derechos humanos, sexuales y reproductivos de los estudiantes de preescolar, básica y media, particularmente, las relacionadas con acoso escolar y violencia escolar incluido el que se pueda generar a través del uso de la internet, según se defina en la ruta de atención integral para la convivencia escolar.

6. Identificar y fomentar mecanismos y estrategias de mitigación de todas aquellas situaciones y conductas generadoras de situaciones de violencia escolar. (...).

Entre de las funciones que la ley le asigna al Comité Nacional de Convivencia Escolar en el artículo 8, vale la pena mencionar las siguientes:

3. Armonizar y articular las acciones del Sistema Nacional con las políticas nacionales, sectoriales, estrategias y programas relacionados con la construcción de ciudadanía, la convivencia escolar y los derechos humanos, sexuales y reproductivos y la prevención y mitigación de la violencia escolar. (...)

8. Promover y liderar estrategias y acciones de comunicación, que fomenten la reflexión sobre la convivencia escolar, la prevención, mitigación, y atención del acoso escolar, la violencia escolar y la disminución del embarazo en la adolescencia, la 
divulgación de la presente ley y de la Ruta de Atención Integral para la Convivencia Escolar, vinculando a los medios de comunicación nacional, regional y comunitarios.

9. Coordinar la creación de mecanismos de denuncia y seguimiento en internet, redes sociales y demás tecnologías de la información a los casos de ciberbullying (...)".

Otra de las novedades de la ley en su artículo 13 es la creación en las instituciones educativas del Comité Escolar de Convivencia, ente que incluirá a representantes de los profesores, de los estudiantes, de los padres de familia, entre otros, y que tendrá dentro de sus funciones:

1. Identificar, documentar, analizar y resolver los conflictos que se presenten entre docentes y estudiantes, directivos y estudiantes, entre estudiantes y entre docentes.

2. Liderar en los establecimientos educativos acciones que fomenten la convivencia, la construcción de ciudadanía, el ejercicio de los derechos humanos, sexuales y reproductivos y la prevención y mitigación de la violencia escolar entre los miembros de la comunidad educativa.

3. Promover la vinculación de los establecimientos educativos a estrategias, programas y actividades de convivencia y construcción de ciudadanía que se adelanten en la región y que respondan a las necesidades de su comunidad educativa.

4. Convocar a un espacio de conciliación para la resolución de situaciones conflictivas que afecten la convivencia escolar, por solicitud de cualquiera de los miembros de la comunidad educativa o de oficio cuando se etime conveniente en procura de evitar perjuicios irremediables a los miembros de la comunidad educativa. El estudiante estará acompañado por el padre, madre de familia, acudiente o un compañero del establecimiento educativo.

5. Activar la Ruta de Atención Integral para la Convivencia Escolar definida en el art\{iculo 29 de esta ley, frente a situaciones específicas de conflicto, de acoso escolar, frente a las conductas de alto riesgo de violencia escolar o 
de vulneración de derechos sexuales y reproductivos que no pueden ser resueltas por este comité de acuerdo con lo establecido en el manual de convivencia, porque trascienden del ámbito escolar, y revisten las características de la comisión de una conducta punible, razón por la cual deben ser atendidos por otras instancias o autoridades que hacen parte de la estructura del Sistema y de la Ruta.

6. Liderar el desarrollo de estrategias e instrumentos destinados a promover y evaluar la convivencia escolar, el ejercicio de los derechos humanos, sexuales y reproductivos.

7. Hacer seguimiento al cumplimiento de las disposiciones establecidas en el manual de convivencia, y presentar informes a la respectiva instancia que hace parte de la estructura del Sistema Nacional de Convivencia Escolar y Formación para los Derechos Humanos, ls Educación para la Sexualidad y la Prevención y Mitigación de la Violencia Escolar, de los casos o situaciones que haya conocido el comité.

8. Proponer, analizar y viabilizar estrategias pedagógicas que permitan la flexibilización del modelo pedagógico y la articulación de diferentes áreas de estudio que lean el contexto educativo y su pertinencia en la comunidad para determinar más y mejores maneras de relacionarse en la construcción de la ciudadanía (...)”.

A continuación la ley le asigna responsabilidades a los diferentes organismos dentro del Sistema Nacional de Convivencia Escolar y Formación para los Derechos Humanos, la Educación para la Sexualidad y la Prevención y Mitigación de la Violencia Escolar, el Ministerio de Educación (artículo 15), la Secretarías de Educación de las entidades territoriales (artículo 16), de los establecimientos educativos (artículo 17), de los rectores o directores de estos(artículo 18) y de los docentes (artículo 19). Posteriormente, la ley establece la necesidad de ajustar los proyectos pedagógicos con el fin de que reflejen "la vivencia y práctica de los derechos humanos en la cotidianidad escolar, cuyo objetivo es la transformación de los ambientes de aprendizaje, donde los conflictos se asumen como oportunidad pedagógica que permite su solución mediante el diálogo, la concertación y el reconocimiento a la diferencia para que los niños, niñas y adolescentes desarrollen competencias para desempeñarse como sujetos activos de derechos en el contexto escolar, 
familiar y comunitario. Para esto el proyecto pedagógico enfatizará en la dignidad humana, los derechos humanos y la aceptación y valoración de la diversidad y las diferencias" (artículo 20).

En cuanto a los manuales de convivencia de las instituciones educativas, un tema que ha generado una gran atención mediática en el último tiempo, resulta pertinente recordar las obligaciones que la Ley de Convivencia Escolar señaló al respecto. Así, dice la normatividad que "los manuales de convivencia deben identificar nuevas formas y alternativas para incentivar $y$ fortalecer la convivencia escolar y el ejercicio de los derechos humanos, sexuales y reproductivos de los estudiantes, que permitan aprender del error, respetar la diversidad y dirimir los conflictos de manera pacífica, así como de posibles situaciones $y$ conductas que atenten contra el ejercicio de sus derechos" (artículo 21). (Negrilla fuera de texto). La ley reconoce el rol de las familias como parte de la comunidad educativa y le asigna responsabilidades dentro del marco del Sistema Nacional de Convivencia Escolar (artículo 22).

Finalmente podemos afirmar que la herramienta más importante que concibió la ley para ejecutar su contenido, fue la creación de la Ruta de Atención Integral para la Convivencia Escolar, que no es otra cosa que un documento mediante el cual se definen "los procesos y los protocolos que deberán seguir las entidades e instituciones que conforman” el Sistema Nacional de Convivencia Escolar, en aquellos casos y situaciones "en que se vea afectada la convivencia escolar y los derechos humanos, sexuales y reproductivos de los estudiantes de las instituciones educativas, articulando una oferta de servicio ágil, integral y complementario" (artículo 29). Esta deberá contener como mínimo cuatro componentes: promoción, prevención, atención y seguimiento.

El de promoción deberá centrarse en el ejercicio de los derechos humanos, sexuales y reproductivos y en el desarrollo de competencias para lograrlo. Se definen los criterios de convivencia a seguir por parte de los miembros de la comunidad educativa, así como los mecanismos y las instancias participativas y se determina la calidad del clima escolar. El componente de prevención, por su parte, se desarrollará mediante la implementación de un proceso continuo de formación integral en los niños y adolescentes, con el fin de

disminuir en su comportamiento el impacto de las condiciones del contexto económico, social, cultural y familiar. Incide sobre 
las causas que puedan potencialmente originar la problemática de la violencia escolar, sobre sus factores precipitantes en la familia y en los espacios sustitutivos de vida familiar, que se manifiestan en comportamientos violentos que vulneran los derechos de los demás, y por lo tanto quienes los manifiestan están en riesgo potencial de ser sujetos de violencia o de ser agentes de la misma en el contexto escolar" (artículo 30).

La atención, como parte de la Ruta de Atención Integral, pretende desarrollar diversas estrategias que permitan brindarles asistencia a los niños, adolescentes, jóvenes, padres, acudientes y educadores, "de manera inmediata, pertinente, ética e integral, cuando se presente un caso de violencia o acoso escolar o de comportamiento agresivo que vulnere los derechos humanos, sexuales y reproductivos (...) Este componente involucra a actores diferentes a los de la comunidad educativa cuando la gravedad del hecho denunciado, las circunstancias que lo rodean o los daños físicos y psicológicos de los menores involucrados sobrepasan la función misional del establecimiento educativo"(artículo 30). Cuando sea necesaria la activación del componente de atención, esta será realizada por el Comité de Convivencia Escolar, debiéndose tener en cuenta lo siguiente (artículo 31):

1. La puesta en conocimiento de los hechos por parte de las directivas, docentes y estudiantes involucrados.

2. El conocimiento de los hechos a los padres de familia o acudientes de las víctimas y de los generadores de los hechos violentos.

3. Se buscarán las alternativas de solución frente a los hechos presentados procurando encontrar espacios de conciliación, cuando proceda, garantizando el debido proceso, la promoción de las relaciones participativas, incluyentes, solidarias, de la corresponsabilidad y el respeto de los derechos humanos.

4. Se garantice la atención integral y el seguimiento pertinente para cadacaso (...)"

Por último, el componente de seguimiento implica el reporte oportuno de la información al Sistema Nacional de Convivencia Escolar según el estado en el que se encuentre el caso de atención que hubiese sido reportado. 
La ley termina con un capítulo dedicado a las sanciones (artículos 35 y 36) e infracciones administrativas (artículo 37) a que haya lugar por el incumplimiento de la ley, así como de una serie de incentivos, mediante los cual el Ministerio de Educación puede reconocer la labor de una entidad educativa por su impacto positivo en la implementación de las estrategias propuestas por la normativa (artículo 39). De igual manera, se señalan sanciones para las instituciones educativas privadas (artículo 36) y las faltas disciplinarias de los docentes y directivos docentes (artículo 38).

Podemos concluir reconociendo el valor de la Ley de Convivencia Escolar, que sus buenas intenciones y su pertinencia deben ser destacados. Se trata de un esfuerzo por recoger las recomendaciones realizadas por la Corte Constitucional en la sentencia T-905 de 2011 con ponencia del Magistrado Jorge Iván Palacio Palacio, y debe resaltarse el carácter multipartidista de la ley, la cual contó mayoritariamente con el apoyo de las fuerzas políticas representadas en el Congreso. Sin embargo, y como veremos a continuación, su aplicación ha sido limitada y se requiere mayor compromiso por parte de los agentes del Sistema Nacional de Convivencia Escolar, para darle cumplimiento a la normativa, buscando generar una cultura de rechazo a cualquier forma de violencia escolar, así como a la protección y defensa de los derechos humanos, sexuales y reproductivos dentro del ámbito escolar.

Sentencia T-365 de 2014. Magistrado Ponente: Nilson Pinilla Pinilla.

Esta sentencia de la Corte hace parte de una subregla jurisprudencial que se ha encargado de desarrollar el tema de la cibercensura o matoneo virtual. Debido a las particularidades propias de ese fenómeno, y a que ha desbordado el ámbito meramente escolar, su estudio a profundidad se realizará en un trabajo posterior. Sin embargo, a manera de introducción, y tratándose de un caso cuyos hechos se desarrollaron en un ambiente escolar, ha sido incluida en el presente documento.

Los hechos del caso la Corte los resume de la siguiente manera:

5.1. Bianca, en representación de Filipo, su hijo menor de edad, incoó esta acción de tutela contra el Colegio AA, aduciendo vulneración a los derechos fundamentales de Filipo a la dignidad, la salud, el buen nombre y la honra, por cuanto sus compañeros de grado crearon un grupo en Facebook, a través del cual divulgaban "información denigrante e intimidatoria" hacía 
su hijo, ante lo cual solicitó que el colegio accionado ordenara a los padres de familia de los estudiantes en cuestión, que les prohibieran el maltrato, en cualquiera de sus formas y se les exigiera un trato respetuoso hacia Filipo, desactivando el grupo social electrónico.

Durante el trámite de la tutela, la Corte decidió suspender los términos del presente proceso, e invitó al Dr. Enrique Chaux, profesor asociado del Departamento de Psicología de la Universidad de los Andes y Director del Programa Multi-Componente Aulas en Paz y del grupo de investigación Agresión, Conflictos y Educación para la Convivencia, con el fin de que emitiera un concepto sobre el asunto objeto de revisión. Si bien la opinión del Dr. Chaux no es vinculante, vale la pena resaltar el interés de la Corte por escuchar voces autorizadas en la materia, toda vez que se trata de uno de los profesionales más reconocidos en Colombia en el trabajo e investigación de la convivencia y el acoso escolar o censura. $\mathrm{Su}$ intervención tiene un valor doctrinal importante para el entendimiento del fenómeno de la cibercensura.

Para la Corporación resulta evidente que uno de los problemas que ha venido en aumento en los últimos años, como consecuencia del avance de las nuevas tecnologías y de las redes sociales, es el acoso escolar.

Bajo el orden constitucional vigente, toda persona, en especial los menores de edad, tiene derecho a que se le proteja del llamado acoso escolar o matoneo (censura), por ser formas expandidas de atentar contra su honra y dignidad. Las tecnologías de la información han conllevado un impacto negativo por la facilidad para que crezca este tipo de conductas, en intensidad y nocividad, al potenciar el daño causado". La Corte cita a la organización canadiense Public Safety Canada, la cual define el ciberbullying como "el uso de nuevas tecnologías de la información y la comunicación para amenazar físicamente, asediar verbalmente o excluir socialmente a un individuo de un grupo.

Durante el trámite de la Tutela, la Corporación pudo constatar que el grupo en la red social Facebook, mediante el cual se estaba agrediendo al menor, había sido desmontado, ante la solicitud de amparo por parte de la madre del menor. "De otra parte, mediante carta de febrero 21 de 2014, Bianca comunicó a la Corte que "puede darse por superado el caso particular de mi hijo, al que observo en excelentes condiciones físicas y emocionales, gracias a la oportuna y efectiva intervención de la 
Corte Constitucional y el especial interés para intervenir, asesorar y acompañarnos en el restablecimiento de los derechos expresados en la tutela interpuesta", manifestando también su "gratitud y sincera felicitación a la gestión de quienes intervinieron y deseando que todos los jóvenes que pasan por estas situaciones tengan el mismo apoyo" (f. 49 ib., no está en negrilla en el texto original). Por ello, esta Corte encuentra que la acusada vulneración de derechos fundamentales ha cesado".

La Corte reitera la jurisprudencia sentada en las sentencias T-917 de 2006 con ponencia del Magistrado Manuel José Cepeda Espinosa y la T905 de 2011 con ponencia del Magistrado Jorge Iván Palacio Palacio. Al resolver el caso concreto, y como parte de una de las órdenes emitidas, replica la realizada en la T-905/2011, mediante la cual se insta

al Ministerio de Educación Nacional y al Instituto Colombiano de Bienestar Familiar, ICBF, para que coordinadamente y en el ámbito propio de sus respectivas funciones, formulen y desarrollen una política general, para precaver, detectar oportunamente, atender y proteger a quienes padezcan hostigamiento, acoso o matoneo escolar, incluyendo el llamado "ciber matoneo" o "cyberbullying", lo que realizarán sistemáticamente y con todas las dependencias territoriales con competencias educacionales, de donde irradie hacia los centros de educación, desde la preescolar, para la actualización de todos los manuales de convivencia, siempre en procura de esculpir desde la niñezuna sólida cultura de paz".

No deja de sorprender que la Corte se vea obligada a realizar dicha invitación, máxime cuando se trata de una providencia posterior a la expedición de la Ley 1620 de 2013. Esto confirma lo mencionado en el aparte anterior, en cuanto a que la aplicación de la normativa contenida en la Ley de Convivencia Escolar, no había sido aplicada. No obstante, el presente caso se prolongó desde antes de la expedición de la ley y hasta después de dicho hecho, lo que podría de alguna manera justificar dicho pronunciamiento.

Sentencia T-563 de 2014. Magistrada Ponente: Gloria Stella Ortiz Delgado.

En la presente Tutela se resuelve un caso en el que se revisa la negativa de una Entidad Promotora de Salud de realizar un procedimiento quirúrgico a un menor, por considerarlo de carácter estético y no estar incluido en el 
Plan Obligatorio de Salud. La madre del menor alega que esa negativa viola los derechos fundamentales ya que debido a su condición física se le han "causado problemas psicológicos como baja autoestima, debido al acoso escolar que sufre dentro de la institución educativa en la que estudia. Por lo anterior, la psicóloga recomendó se realice la "cirugía otoplastia bilateral”, ordenada por el médico tratante de la EPS ",4.

Como consecuencia de lo anterior, la Corte decide resolver, entre otros, el siguiente problema jurídico: “¿La omisión en la práctica del procedimiento requerido por el niño, conlleva al detrimento de la vida en condiciones dignas, atendiendo al acoso escolar al cual se encuentra sometido dentro de la institución educativa a la que pertenece? (...).

Este es el primer pronunciamiento en el que la Corte expresamente reconoce la existencia de la Ley de Convivencia Escolar, cuando afirma que recientemente y debido a "las circunstancias de violencia generadas dentro de los planteles educativos, se expidió la Ley 1620 de 2013, con el propósito de establecer mecanismos de prevención, protección, detención temprana y denuncia sobre la violencia escolar, entre las que se encuentra la "censura". Define la censura como "un fenómeno social y una forma de maltrato específico, intencional, perjudicial, reiterado, continuo, discriminatorio y persistente de un estudiante o grupo de estudiantes hacia otro compañero, que generalmente se presenta en el ámbito escolar","

De igual manera, sostuvo que "el acoso escolar entre otras conductas se presenta con el hostigamiento, intimidación, maltrato, violencia, exclusión social y discriminación que sufre un niño en el entorno escolar por parte de uno o varios compañeros. De este modo, a el acoso puede provenir la violencia física, verbal, simbólica y en particular emocional, que atenta contra la dignidad del menor de edad y ocurre de manera i) intencional ii) reiterada y iii) continua". Esto hace que en consideración de la Corte, "los menores tienen derecho a que se les proteja del acoso escolar, por constituir una forma expandida de afectación de la honra y dignidad, y una conducta que impacta negativamente en el desarrollo integral de la comunidad estudiantil”.

4 Consideración 2

5 Consideración 3. Para formular esta definición, la Corte se apoya en los siguientes materiales: Cfr. "Bullying at schooll, what we know and we can do", Dan Olweus, Cambridge, Massachusetts. "Múltiples Perspectivas Sobre un Problema Complejo: Comentarios Sobre Cinco Investigaciones en Violencia Escolar", Enrique Chaux, Universidad de los Andes. "School bullying”, Alana James, University of London. "Bullying prevention and intervention: infromation for educators", Philip J. Lazarus, Florida International University and William Pfohl, Western Kentucky University. 
Sentencia T-478 de 2015. Magistrada Ponente: Gloria Stella Ortiz Delgado.

Se trata del tristemente célebre caso de Sergio Urrego, el joven de 17 años que se quitó la vida como consecuencia de un caso de matoneo o censura, perpetrado por parte de las directivas del Colegio al cual pertenecía, luego de que un profesor de la institución encontrara una fotografía de él besándose con otro alumno del colegio, en el celular que le había sido decomisado a una compañera suya. El análisis del caso es extenso y busca escuchar a todos los actores involucrados dentro del Sistema Nacional de Convivencia Escolar. El presente caso desnudó crudamente la falta de aplicación de la Ley de Convivencia Escolar, que precisamente se había expedido con el fin de prevenir situaciones como esta. Debido a lo extenso de la sentencia, en este trabajo únicamente nos referiremos a los argumentos esgrimidos por la Corporación, para resolver el siguiente problema jurídico:

“¿Constituye una situación de acoso escolar por orientación sexual por parte del colegio acusado y de violación a otros derechos fundamentales el iniciar un proceso disciplinario a una pareja del mismo sexo por considerar que realizaban manifestaciones de afecto obscenas y vulgares e incurrir en una serie de medidas posteriores que pudieron ser un factor determinante en el suicidio de uno de ellos, cuando para el colegio las actividades desplegadas por la institución no fueron más que consecuencia de la aplicación del Manual de Convivencia, del mal comportamiento del estudiante fallecido y de su hogar disfuncional?"

Lo primero que nos interesa es conocer la definición que la Corte adopta sobre la censura o acoso escolar, "respaldada por la literatura científica sobre la materia, indica que este fenómeno (conocido también como acoso escolar o "bullying") es la agresión repetida y sistemática que ejercen una o varias personas contra alguien que usualmente está en una posición de poder inferior a la de sus agresores. Esta deliberada acción sitúa a la víctima en una posición en la que difícilmente puede escapar de la agresión por sus propios medios". Así las cosas, la Corte afirma que "la intimidación es un abuso que está asociado directamente a un desequilibrio de poder entre quien agrede y quien es agredido" y continúa diciendo que "no puede ser resuelta a través de una mediación de pares, sino que se requiere de una acción institucional de prevención y 
acompañamiento que permita superar una situación de esta naturaleza. Incluso, esta acción institucional debe buscar prevenir las graves consecuencias que la afectación a la intimidad tiene en la vida de las personas".

Por primera vez la Corte hace referencia a los diferentes roles que desde la academia se han asignado a quienes participan en una situación de acoso escolar o bullying, identificándolos como el agresor, la víctima y los espectadores ${ }^{6}$. Adicionalmente la Corporación se pregunta si "¿el acoso escolar es un fenómeno que solo ocurre entre pares (es decir entre estudiantes) o si las agresiones pueden provenir también, por ejemplo, de las autoridades de las instituciones educativas?"(Negrilla fuera de texto). La respuesta a la que llega la sentencia es que desafortunadamente "la intimidación escolar puede tener características estructurales que se desprenden de políticas o prácticas discriminatorias auspiciadas por las directivas de un colegio" (Negrilla fuera de texto). La sentencia admite como ciertos los datos recogidos en un estudio del Dr. Enrique Chaux y admite su preocupación al observar "como el acoso escolar es un fenómeno extendido en el sistema educativo colombiano" y que no se trata de "una práctica aislada en el sistema educativo en Colombia. Por el contrario, es un fenómeno de características masivas que tiene causas estructurales relacionadas con estereotipos alrededor del concepto de debilidad y las formas de obtener poder".

En otro aparte, la Corte realiza un análisis de lo que denomina los "Problemas estructurales en materia de resolución de conflictos por acoso escolar en razón de diferencias en la identidad de género o la orientación sexual en el sistema educativo colombiano ". Lo primero que hace la sentencia en este punto es presentar un cuadro explicativo de "las características generales del funcionamiento del sistema, señalando en términos básicos, la distribución de competencias entre los órganos nacionales y del orden departamental y municipal”, basándose en las disposiciones de la Ley 115 de 1994, también conocida como la Ley General de Educación. Según la Corte, "una importante adición a este sistema de inspección y vigilancia, es la recién sancionada Ley de Convivencia Escolar -Ley 1620 de 2013- y su decreto reglamentario Decreto 1965 de 2013-. Dicha norma, explícitamente reconoce que uno

6 Cuadro 2. Cuadro elaborado por el despacho de la magistrada sustanciadora a partir de información recogida en: CHAUX, Enrique. Educación, convivencia y agresión escolar. Ediciones Uniandes. Bogotá (2012), y COLLELL i CARALT, Jordi y ESCUDÉ MIQUEL, Carme. El acoso escolar: un enfoque psicopatológico. En: Anuario de Psicología Clínica de la Salud. Volumen 2 (2006). 
de los retos que tiene el país, está en la formación de sus ciudadanos, para el ejercicio activo de la ciudadanía y de los Derechos Humanos. De esta manera, la norma fue pensada como una política de promoción y fortalecimiento de la convivencia escolar, precisando que cada experiencia que los estudiantes vivan en los establecimientos educativos resulta fundamental para el desarrollo de su personalidad".

No obstante la existencia de mecanismos de Inspección, Vigilancia y Control en el Sistema Educativo, así como una nueva política pública en contra de las prácticas de acoso escolar, censura o matoneo, en la cual se buscaba prevenir precisamente hechos como los presentados en este caso, "resulta notorio, sin embargo, que ninguna de las acciones de detección temprana de las normas en mención, fuera implementada en el presente caso constitucional. De un examen general de las competencias de inspección y vigilancia solo se logra constatar que las entidades involucradas aplicaron sus facultades generales de sanción. Sin embargo, las rutas de acción y las garantías de convivencia escolar no lograron detectar una posible situación de intimidación pues, sencillamente, nunca fueron implementadas por las autoridades competentes". Como se mencionó, este caso puso en evidencia la falta de implementación práctica de la Ley, la cual quedó reducida a letra muerta ante la inoperancia de sus disposiciones. Dice la Corte que debido a lo anterior, "existe un déficit de protección general para las víctimas de acoso escolar ante estas circunstancias, ya que, a pesar de que existe un marco regulatorio claro y una política pública definida desde el 2013, la misma no ha sido implementada con vigor $y$ en casos como el que nos convoca, ni siquiera fue impulsada en momentos concretos" (Negrilla fuera de texto).

Por último, al realizar el análisis del caso concreto la Corte decide analizar "Las fallas estructurales ante el acoso escolar en el sistema educativo colombiano". Allí la Corte reitera que a pesar de que la Ley 1620 de 2013, entre otras cosas, creó

mecanismos de detección temprana, acción preventiva, conciliación y seguimiento a este fenómeno de acoso, sea entre pares o desde una perspectiva institucional, es claro que ninguna autoridad pública o el colegio accionado o alguno de los intervinientes en el proceso, activó dichos procedimientos, con el fin de encontrar una solución consultada, integral y respetuosa de los derechos fundamentales de los jóvenes, en un contexto educativo en el que se deben formar los ciudadanos del mañana. 
En ese orden de ideas, continúa diciendo que en "ningún momento, se observó una actuación coordinada entre las autoridades demandas, para acompañar el proceso de formación de los jóvenes y las dificultades que estaban enfrentando ellos mismos, frente a las presiones institucionales y familiares. Por ello es que la política propuesta puede ser un punto de partida importante para acceder a propuestas concertadas en estos casos". Así mismo, termina afirmando que "existiendo una política pública vigente que pretende ser un instrumento eficaz de convivencia escolar dirigido a la promoción de los derechos humanos, entre los que se encuentra el ejercicio libre de los derechos sexuales y reproductivos y el respeto por la diversidad sexual, es necesario que se intensifiquen los mecanismos administrativos para asegurar la operatividad del sistema, para que casos como estos no vuelvan a ocurrir".

Como consecuencia de los hechos del caso, así como de las consideraciones realizadas por la Corporación a lo largo de la sentencia, la Corte termina concluyendo que

atendiendo el déficit de protección que enfrentan las víctimas de acoso escolar en el país, ante la falta de operatividad de la política pública de convivencia escolar, la Sala le dará al Ministerio de Educación, como ente coordinador de esa, una serie de órdenes tendentes a implementar en un plazo razonable mecanismos de detección temprana, acción oportuna, acompañamiento y seguimiento a casos de acoso escolar. Esto con el fin de evitar que casos tan lamentables como el que se examina en esta oportunidad, vuelvan a ocurrir. No es concebible, dentro de un Estado Social de Derecho, que la trágica muerte de un joven, producto de la incomprensión, sea una nueva razón para reconocer nuestro compromiso en evitar que la realidad masiva, reiterada y estructural de la violación de los derechos fundamentales de las niñas, los niños y los adolescentes en nuestro país, continúe. Es imposible aspirar a una sociedad robusta, deliberativa, plural y democrática si nuestros ciudadanos son formados a partir del sobresalto y la incomprensión.

Vale la pena nuevamente resaltar los esfuerzos de la Corte Constitucional por entender y aportar a la solución del acoso escolar, matoneo o censura, mediante sus pronunciamientos y órdenes que realiza en sus sentencias. Es lamentable que se hubiese tenido que sacrificar la vida de un joven para llamar nuevamente la atención sobre la falta de aplicación de la 
normativa vigente. De igual manera, es penoso, por decir lo menos, descubrir durante el trascurso de la sentencia, el abuso sistemático al que se vio sometido Sergio, por parte de las autoridades del Colegio, las cuales aún después de su muerte, continuaron vulnerando sus derechos fundamentales al buen nombre, filtrando información a los medios de comunicación y realizando todo tipo de acusaciones en contra de un muchacho por sus vinculaciones políticas, su tendencia sexual y las particularidades de su estructura familiar.

A partir de este fallo, y como consecuencia de las órdenes impartidas por la Corte, se inició el proceso por parte del Ministerio de Educación, de los Manuales de Convivencia de las instituciones educativas del país, generando una gran discusión en el país, el cual ha estado desafortunadamente liderado por la desinformación, los prejuicios y la falta de conocimiento del marco legal que aquí se ha buscado presentar. El proceso, liderado por la ya ex Ministra de Educación, no era un capricho o una imposición originada en su propia identidad sexual, sino que se trataba de uno iniciado con mucha anterioridad, avalado por la gran mayoría de las fuerzas políticas del país quienes en su momento apoyaron, aprobaron y expidieron la Ley 1620 de 2013. Es nuestro deseo que este caso, sirva verdaderamente para, como lo afirma la ley, "aprender del error" (artículo 21) y que verdaderamente nunca se vuelva a repetir.

Sentencia T-281A de 2016. Magistrado Ponente: Luis Ernesto Vargas Silva.

Se trata del pronunciamiento más reciente de la Corte Constitucional en abordar el tema del bullying o acoso escolar. En ella se reitera y consolida la jurisprudencia sentada por la Corte citando las sentencias T905 de 2011 (4 veces), T-365 de 2014 (2 veces) y T-478 de 2015 (3 veces). Fue utilizada como "punto nodal", concepto desarrollado por López Medina y que sirve como punto de partida de la investigación jurisprudencial realizada.

Se resuelve una tutela mediante la cual se busca la protección del derecho al debido proceso de un estudiante luego de que la institución educativa resolviera que no debería continuar en el colegio, luego de que este hubiera creado un grupo en una red social en la cual publicó unas fotos de una compañera desnuda. El Colegio alegó que se trató de un proceso "tras desplegar algunas situaciones de incumplimiento de las normas de convivencia escolar". 
Vale la pena rescatar la presentación conceptual que se realiza tanto de censura como de la cibercensura. Sobre el primero, dice la sentencia que estamos ante un concepto que ha sido "definido por la literatura especializada como una agresión que se caracteriza por (i) ser intencional; (ii) envolver un desequilibrio de poder entre un agresor (el cual puede ser individual o grupal) y una víctima; así como (iii) por ser repetitiva y producir efectos en el transcurso del tiempo. El Bullying se genera a través de insultos, exclusión social, propagación de rumores, entre otras formas, en contextos de confrontación personal (cara a cara) o con palabras escritas, por ejemplo, las empleadas a través de medios de comunicación como internet". Esta última modalidad, el ciberbullying, lo define como

una intimidación a través de mensajes de correo electrónico, servicios de mensajería instantánea, sitios web, o imágenes enviadas a los teléfonos celulares, entre otras formas. Se caracteriza porque (i) se puede desplegar desde al anonimato, lo cual, a su vez; (ii) genera que los victimarios lleguen a tener comportamientos que tal vez no tendrían si las intimidaciones fueran en contextos de confrontación personal; (iii) los medios para generarlo están al alcance en cualquier momento gracias a las facilidades de acceso de los medios electrónicos; (iv) la victima tiene temor de denunciarlo ante eventuales represalias del victimario o porque se le restrinja el uso de su computador o celular y; (v) el número de espectadores que puede conocer el contenido de los mensajes intimidatorios es alto, dada la demanda de usuarios de internet.

Por otra parte, la Corte recuerda la creación legal de los Comités de Convivencia Escolar y su desarrollo reglamentario, contenido en el Decreto 1075 de 2015. Dice el decreto:

Todas instituciones educativas y centros educativos oficiales y no oficiales del país deberán conformar Comité Escolar de Convivencia, encargado de apoyar la labor de promoción y seguimiento a la convivencia escolar, a la educación para el ejercicio de los derechos humanos, sexuales y reproductivos, así como del desarrollo y aplicación del Manual de Convivencia y de la Prevención y Mitigación de la Violencia El respectivo consejo directivo de las referidas instituciones y centros educativos dispondrá de un plazo no mayor a seis (6) meses, contados a partir del 11 de 2013, para conformar el Comité Escolar de 
Convivencia y elaborar su reglamento, el cual deberá hacer parte integral del manual de convivencia.

La Corte encontró que en este "caso la actividad desplegada por el Comité Escolar de Convivencia no se acompasa con la gravedad de los hechos puestos en conocimiento. Dicho Comité fue displicente ya que pese a conocer los hechos concernientes al censura y contemplar un programa de intervención grupal para la formación en derechos humanos y el uso adecuado de las redes sociales en el Colegio, postergó su ejecución bajo el argumento de estar desarrollando otras actividades escolares".

Como consecuencia de ello, la Sala concluyó que "el Comité Escolar de Convivencia del Colegio Tolimense no atendió la gravedad de los hechos puestos en conocimiento sobre el Cyber Bullying del que fuera víctima una de sus estudiantes. Ello por cuanto (i) postergó la ejecución del programa de intervención grupal para la formación en derechos humanos y el uso adecuado de las redes sociales en el Colegio y (ii) incumplió el deber de desarrollar acciones preventivas para mitigar la violencia escolar, pues contempló la creación de dicho programa luego de presentarse los hechos de intimidación escolar por parte del joven Parra Céspedes".

En ese orden de ideas, la Corte le ordenó al Colegio que desarrollara "una política escolar para la oportuna prevención, detección, atención y protección frente al Bullying o el Cyber Bullying, con la finalidad de evitar situaciones similares a las desplegadas por Juan Esteban, o cualquier otra forma de violencia escolar que vaya en detrimento de los derechos fundamentales de los miembros de la comunidad educativa. La política escolar deberá hacer hincapié en la enseñanza y la observancia de los derechos de la mujer a tener una vida libre de violencia y a que se respeten y protejan sus derechos humanos".

En esta sentencia podemos ver cómo, pasados 3 años desde la expedición de la Ley 1620 de 2013, y si bien en el caso concreto se habían cumplido por parte de la Institución Educativa parte de la controversia constitucional parte de sus obligaciones legales de tipo formal, como la creación del Comité de Convivencia Escolar, la misma entidad no le dio prioridad ni relevancia a las situaciones y comportamientos analizados en la presente acción de tutela, pues postergó la ejecución de programas de intervención para la formación en derechos humanos, utilizando una argumento que a todas luces resulta 
inadecuado, como afirmar que se estaban desarrollando otras actividades.

\section{CONCLUSIONES}

Es importante resaltar que en estos 10 años desde que la Corte se pronunció en la sentencia T-917 de 2006 con ponencia del Magistrado Manuel José Cepeda Espinoza, se han presentado significativos e importantes avances en la materia. No obstante lo anterior, sería necio no señalar que dichos avances se han presentado en gran medida por el activismo judicial que ha tenido la Corte y no iniciativa propia de los Gobiernos nacionales, departamentales o distritales, y mucho menos por iniciativa de las entidades educativas del sector público o privado.

De igual manera es necesario hacer énfasis en la falta de voluntad por parte de las entidades que conforman el Sistema Nacional de Convivencia Escolar, pues fue únicamente a partir de la sentencia T-478 de 2015 con ponencia de la Magistrada Gloria Stella Ortiz Delgado, que las entidades del orden nacional, en cabeza del Ministerio de Educación y las demás partícipes del Sistema, se "pusieron las pilas", en el tema de prevención y mitigación de la violencia escolar o censura, y comenzaron a darle aplicación real a la Ley 1620 de 2013, mediante la creación de los Comités de Convivencia Escolar y la aplicación de la Ruta Integral de Atención, concebida como herramienta principal de la Ley. Es desafortunado que haya sido necesario de la constante intervención de la Corte Constitucional para que se cumplieran las obligaciones legales y se pusiera en marcha la política pública de prevención y atención de la violencia y del acoso escolar.

Es verdad que estamos ante un problema mayúsculo y que no será fácil de solucionar. Vemos como las tecnologías de la información y las redes sociales han creado nuevos espacios en los cuales se pueden dar situaciones de acoso, violencia, matoneo y censura, que muchas veces tienen el potencial de trascender al espacio educativo, lo que dificulta su análisis y requiere diferentes acciones por parte de las entidades del Sistema Nacional de Convivencia Escolar. Se requieren acciones coordinadas por parte del Sistema en su conjunto y que cada individuo se eduque y asuma como propia la erradicación de la cultura del acoso escolar.

Es imperioso que se tomen acciones tendentes a promover en los estudiantes y en la comunidad educativa en general un cambio cultural 
de respeto por los derechos humanos, sexuales y reproductivos, que celebre la diversidad, el multiculturalismo, la libertad y los valores democráticos. Es responsabilidad de cada uno de nosotros, como padres, estudiantes, profesores, profesionales, directivos escolares, generar conciencia sobre la importancia de acatar la normatividad y enfrentar el cambio con optimismo y verlo como una oportunidad de educar mejores y más integrales colombianos, que contribuyan a la consolidación de una paz estable y duradera.

Vale la pena hacerle un llamado a todas las entidades, públicas y privadas, así como a las personas que hacen parte del Sistema Nacional de Convivencia Escolar y Formación para los Derechos Humanos, la Educación para la Sexualidad y la Prevención y Mitigación de la Violencia Escolar, sobre la necesidad de desarrollar estrategias pedagógicas diversas que aborden de manera técnica, especializada y novedosa, los tres grandes ejes fundamentales contenidos en la Ley 1620 de 2013. De igual manera, resulta ineludible fortalecer el Sistema de Inspección, Vigilancia y Control del sector educativo, con el fin de que se garantice el cumplimiento de las obligaciones legales de los intervinientes.

Resulta claro que la Corte ha adoptado una definición soportada en la doctrina especializada sobre el fenómeno del acoso escolar o censura. Esa definición implica que estamos en presencia de situaciones de acoso escolar o censura, cuando las conductas o comportamientos son reiterados, tienen la intención de causar un daño, se presentan como consecuencia de un desbalance de poder entre las partes intervinientes. Las estrategias pedagógicas que las Instituciones Educativas deben incluir dentro de sus programas académicos, tienen que estar encaminadas a brindarle herramientas de juicio a los estudiantes sobre las diferentes maneras en las que pueden actuar al estar en presencia de conductas o situaciones de acoso escolar o censura. Entre ellas podemos incluir explicaciones conceptuales sobre las diferencias entre el acoso y el simple conflicto, las diferentes formas en las que este fenómeno puede manifestarse, tales como agresiones físicas, psicológicas, intimidación, ridiculización, difamación, coacción, aislamiento deliberado, entre otros. Así mismo, es necesario que se realicen demostraciones sobre la manera de transmitir mensajes de manera asertiva, así como sobre la importancia de ser espectadores y ciudadanos éticos, que intervengan para frenar situaciones en las que se presentan violaciones de los Derechos Humanos de otros. 
La única manera de combatir la maquinaria y estrategia de desinformación propuesta por algunos sectores ultra conservadores y religiosos que pretenden anular y desmontar los derechos adquiridos por las minorías reconocidos en la Constitución y la jurisprudencia de la Corte Constitucional, así como de los tratados internacionales de derechos humanos, es mediante un esfuerzo mayúsculo de pedagogía sobre los contenidos de la normativa, los objetivos, valores y principios perseguidos por la jurisprudencia, las leyes y reglamentos vigentes que han regulado y desarrollado el sistema de convivencia escolar en nuestro país. Resulta sorprendente que el debate suscitado como consecuencia del cumplimiento de una orden emitida en un fallo de tutela por la Corte Constitucional al Ministerio de Educación, hubiese llegado a generar la polarización tan profunda que se generó en el país, más cuando hacía relativamente poco había sido presentado, discutido y aprobado, el proyecto de ley que se convertiría en la Ley de Convivencia Escolar, cuyos ponentes son miembros de los partidos Conservador (2) y Liberal (1). Las familias hacen parte del Sistema, y como tal tienen derechos y obligaciones dentro del mismo, sus creencias, valores y principios deben ser respetados, sin que ellos deban serles impuestos u otros miembros de la comunidad que promulguen confesiones o valores diferentes.

Es evidente que el acoso escolar o censura es un problema generalizado en las Instituciones Educativas del país, y que tiende a ser minimizado, invisibilizado y de plano negado, por la mayoría de las directivas y profesores de las entidades. Muchas veces estas personas consideran que los casos que se dan en sus instituciones, o bien no son tan graves como los estudiados por la Corte, o se trata de simples hechos aislados que no son recurrentes y que por lo tanto el fenómeno como tal no es un problema prevalente dentro de sus Colegios. Mientras prevalezca esta mentalidad, será muy difícil poner en marcha los mecanismos que la ley estableció para promover el desarrollo de ciudadanos respetuosos de los derechos humanos, sexuales y reproductivos, que rechacen la violencia y la agresión como formas de resolver conflictos, que demuestren empatía y solidaridad para con personas en situaciones de vulnerabilidad y que sepan comunicar mensajes contundentes y asertivos que permitan poner fin a conductas o situaciones de acoso escolar o censura en sus colegios.

Como parte del trabajo que se buscará desarrollar hacia el futuro, está la necesidad de realizar otros trabajos similares al realizado en el presente documento, con el fin de abordar y analizar otras líneas jurisprudenciales que sirvan de fundamento para el desarrollo e implementación de 
estrategias pedagógicas diversas que contribuyan en el cumplimiento de los objetivos generales de la Ley de Convivencia Escolar. En ese orden de ideas, se realizarán trabajos específicos en cibercensura, Educación para la Salud Sexual y Reproductiva en el Sistema Legal Colombiano; el consentimiento de las personas en situación de discapacidad frente a sus derechos sexuales y reproductivos; procedimientos quirúrgicos estéticos en menores de edad; prevención de la violencia en contra de la mujer; entre otros.

\section{REFERENCIAS BIBLIOGRÁFICAS}

Olweus, D. (1998). Conductas de acoso y amenaza entre escolares. Madrid: Ediciones Morata.

Castillo-Pulido, L. (2001). El acoso escolar: De las causas, origen y manifestaciones a la pregunta por el sentido que le otorgan los actores. Magis Revista Internacional de Investigación en Educación, 4 (8) p. 415-418.

Chaux, E. (2011). Variables socio-económicas, sociopolíticas y socioemocionales que explican la intimidación escolar. Un análisis multinivel a nivel país. Bogotá: Centro de Convenciones Jiménez de Quesada

Chaux, E. (2012). Educación, convivencia y agresión escolar. Bogotá: Ediciones Uniandes.

Listado de Sentencias:

Corte Constitucional de Colombia. (2006). Sentencia T-917. M.P: Manuel José Cepeda Espinosa.

Corte Constitucional de Colombia. (2011). Sentencia T-905. M.P: Jorge Iván Palacio Palacio.

Corte Constitucional de Colombia. (2014). Sentencia T-365. M.P: Nilson Pinilla Pinilla.

Corte Constitucional de Colombia. (2014). Sentencia T-563. M.P: Gloria Stella Ortiz Delgado. 
Corte Constitucional de Colombia. (2015). Sentencia T-478. M.P: Gloria Stella Ortiz Delgado.

Corte Constitucional de Colombia. (2016). Sentencia T-281A. M.P: Luis Ernesto Vargas Silva. 